\title{
Physical Activity Attenuates Increased Obesity Risk Associated with the High-Risk Genotype of the FTO Gene in a UK Adult Population
}

\begin{abstract}
Introduction

Obesity is an international epidemic. Worldwide, 1.9 billion adults are overweight, and 650 million adults are obese. Obesity has many chronic comorbidities, including diabetes and cardiovascular disease. Obesity's cause is positive energy balance involving environmental and biological factors.

Genome-Wide Association Studies (GWAS) suggest that the A/T expression of Single Nucleotide Polymorphism (SNP) rs9939609 within the Fat-Mass and Obesity-Associated (FTO) gene significantly influences adiposity. The high-risk A allele is associated with increased Body Mass Index (BMI) $\left(0.36 \mathrm{~kg} / \mathrm{m}^{2}\right.$ per A allele) and a preference for increased fat and protein intake. FTO encodes for 2-oxoglutarate-dependant-nucleic-acid-demethylase, which is involved in energy homeostasis and lipolysis. Previous studies show that a high physical activity level (PAL) attenuates the increased BMI risks associated with the high-risk allele. However, results have been contradictory. This study aims to elucidate the links between FTO SNP rs9939609, PAL, BMI and food preference.
\end{abstract}

\section{Methods}

An observational cross-sectional study including $37 \mathrm{UK}$ adults was performed. Participant height, weight and body fat $\%$ (BF $\%$ ) (using Bioelectrical Impedance Analysis) were measured. A food frequency questionnaire was completed, a saliva sample was collected for DNA analysis and PAL was assessed using participants active hours.

A two-way MANOVA was performed to determine differences in BMI and BF\% between FTO rs9939609 genotypes and PALs. A Chi-squared test was performed to determine potential genotype associations with food preference.

\section{Results}

Participant BMI was significantly higher in AA participants compared to AT $\left(3.99 \mathrm{~kg} / \mathrm{m}^{2} \pm 1.69, \mathrm{p}=0.027\right)$ and TT $\left(3.7 \mathrm{~kg} / \mathrm{m}^{2} \pm 1.73\right.$, $\mathrm{p}=0.043)$ participants. Participant BMI was higher in low PAL participants compared to medium PAL participants $\left(4.62 \mathrm{~kg} / \mathrm{m}^{2} \pm\right.$ $1.35, \mathrm{p}=0.002)$. Genotype did not significantly influence participant $\mathrm{BF} \%(\mathrm{p}=0.281)$.

A combination of PAL and genotype was significantly associated with BMI and BF\% $(p=0.009)$. BMI was significantly increased in AA participants compared to TT participants $\left(7.15 \mathrm{~kg} / \mathrm{m}^{2} \pm 2.68, \mathrm{p}=0.013\right)$ and AT participants $\left(8.15 \mathrm{~kg} / \mathrm{m}^{2} \pm 2.77, \mathrm{p}=0.007\right)$ with low PAL. This difference was not seen as PAL increased.

No significant associations were found between genotype and food preference $(p=0.717)$.

Discussion

This study suggests that combining genotype and PAL significantly influences BMI and BF\%. Participants homozygous for the risk allele AA with low PAL have significantly higher BMI than AT/TT participants with low PAL. However, the obesogenic effects of the risk genotype are diminished as PAL increases. This suggests that PAL attenuates genetic predisposition for obesity associated with the FTO rs9939609 A allele. These results may be applied in obesity intervention programmes particularly for encouragement of genetically predisposed individuals.

\section{Conflict of Interest}

There is no conflict of interest 